\title{
Two-Dimensional Reduced Theory and General Static Solution for Uncharged Black $p$-Branes
}

\author{
Marco Cavaglià* \\ Department of Physics and Astronomy \\ Tufts University \\ Medford, MA 02155 U.S.A.
}

June 13, 2021

\begin{abstract}
We derive a two-dimensional effective dilaton - gravity - matter action that describes the dynamics of an uncharged black $p$-brane in $N$ dimensions. We show that this effective theory is completely integrable in the static sector and establish its general static solution. The solution includes, as a particular case, the boost symmetric $p$ brane solution investigated in Ref. 9].
\end{abstract}

\section{Introduction}

Recently a large amount of time has been devoted to the investigation of solutions of Einstein and/or dilaton gravity in dimensions other than four [1].

In lower dimensional gravity the study of solutions in two- and three- dimensions has received a lot of attention because of its connection with string

*New address: Max-Planck-Institut für Gravitationsphysik, Albert-Einstein-Institut, Schlaatzweg 1, 14473 Potdsam, Germany 
theory [3], dimensionally reduced models (minisuperspaces and midisuperspaces) [4] and the black hole physics [5]. Lower dimensional models may further provide some insight into the difficult challenge of quantizing gravity theories in the (more physical) four dimensional case [6]. Hence, many $0+1,1+1$ and $2+1$ integrable models have been analyzed and solved in the literature, both from the classical and quantum points of view [6, 7].

On the other hand, the investigation of solutions in more than four dimensions has also raised considerable interest [2, 8]. The most promising candidate for a unified theory of all interactions, the so called M-theory, is indeed naturally formulated in a $N>4$ dimensional spacetime so it seems natural to query how extra dimensions are affecting the four-dimensional world; it is well know, for instance, that in $N>4$ dimensions event horizons may have interesting non trivial topologies [9].

In this context many papers have been devoted to the discussion of uncharged and charged (black) p-branes [9, 10, 11], that are essentially a Ddimensional black hole times a $p$-dimensional flat Euclidean space. The analysis of black $p$-branes is indeed relevant both to supergravity theories [10] and to cosmology, since $p$-branes can also be seen as topological defects [9].

The aim of this paper is to give a contribution to the discussion of both lower dimensional models and pure gravity $p$-brane solutions. Starting from a generic ansatz in $N$ dimensions with topology D-hole $\times p$-brane we reduce the $N$ dimensional Einstein-Hilbert action to a two-dimensional dilaton gravity action plus a massless scalar field with a non-trivial coupling to the dilaton. The static sector of this effective theory turns out to be completely integrable and we establish the general static solution of the system. We believe that the discussion of this model is important at least for two reasons: first it represents an example of an integrable dilaton - gravity - matter model in $1+1$ dimensions with a non trivial coupling between the dilaton and the scalar field. To our knowledge there are no other integrable models with these characteristics in the literature [12]. Further, the general static solution of this system is also the general static solution in $N$ dimensions of a generic uncharged black $p$-brane with dimensions $p \leq N-2$. This solution includes, as a particular case, the generic uncharged solution with boost symmetry of Ref. [9]. We will also see that there are indeed two sets of boost symmetric $p$-branes corresponding to the solutions of Ref. [9] and that these are related by a duality-like relation.

The paper is thus divided in two steps. We first derive the effective two- 
dimensional action and write the equations of motion. Then we integrate the equations of motion in the static sector and discuss their connection with the boost symmetric solutions of Ref. [9].

\section{Two-Dimensional Effective Theory}

Our starting point is the Einstein-Hilbert Lagrangian in $N \geq 4$ dimensions. We consider a multidimensional manifold endowed with a pseudoRiemannian metric of the form

$$
d s^{2}=g_{\mu \nu}(y) d y^{\mu} d y^{\nu}+f(y) \delta_{i j} d z^{i} d z^{j}
$$

where $\mu, \nu=0, \ldots N-p-1, i, j=N-p, \ldots N-1$, and $g_{\mu \nu}$ is a pseudoRiemannian spherically symmetric metric in $N-p$ dimensions. The ansatz (11) includes as a particular case the boost symmetric metric of a $p$-brane of Ref. 9

The model can be reduced to a two-dimensional effective theory using a suitable ansatz for the line element (11). Write (1) in the form

$$
d s^{2}=\phi^{\frac{1}{p+q}-1} h_{a b}(x) d x^{a} d x^{b}+\phi^{\frac{2}{p+q}} e^{\psi} d \Omega^{(q)}+\phi^{\frac{2}{p+q}} e^{-\frac{q}{p} \psi} \delta_{i j} d z^{i} d z^{j},
$$

where $\phi$ and $\psi$ are two functions of $x, d \Omega^{(q \equiv N-p-2)}$ is the metric of the $q$ dimensional unit sphere, and $h_{a b}(a=0,1)$ is a generic two-dimensional pseudo-Riemannian metric. Using (2) the $N$-dimensional Einstein-Hilbert action reduces to the form (we neglect surface terms that are irrelevant for the present discussion)

$$
S=\frac{1}{\kappa} \int d^{2} x \sqrt{-h}\left[\phi R^{(2)}(h)+Y(\phi, \psi)+Z(\phi)(\nabla \psi)^{2}\right],
$$

where $R^{(2)}(h)$ is the two-dimensional Ricci scalar and $Y$ and $Z$ are the two functionals

$$
Y(\phi, \psi)=q(q-1) e^{-\psi} \phi^{-\frac{1}{p+q}}, \quad Z(\phi)=-\frac{q}{4 p}(p+q) \phi
$$

$\kappa$ is a constant factor equal to $16 \pi G /\left(V_{b}^{(p)} V_{s}^{(q)}\right)$ where $V_{b}^{(p)}$ and $V_{s}^{(q)}$ are respectively the volume of the unit $p$-brane and of the unit $q$-sphere. Without 
loss of generality, we will set $\kappa=1$ and thus in the following (3) must be considered an action density in $p$-brane space.

The theory described by the action (3) is an example of a two-dimensional dilaton - gravity - matter model with a non trivial coupling between the matter field and the dilaton field since $\partial_{\phi} Z \neq 0$ [12]. All fields in (3) represent gravitational degrees of freedom of the $N$-dimensional spacetime. The model can be of course extended to include a genuine matter field, as the dilaton, and the Maxwell field. For instance, working in the Einstein frame the dilaton term is

$$
S_{d}=\int d^{2} x \sqrt{-h}\left[-\frac{4}{p+q} \phi(\nabla \chi)^{2}\right],
$$

where, of course, the dilaton field $\chi$ depends only on the $x$ coordinates. The dilaton contributes to the action essentially as the field $\psi$. Solving the equation of motion for the dilaton, one can see that its contribution reduces to a potential term $V(\phi)$ in the action (3). Thus the inclusion of the dilaton, as well as the Maxwell field, modifies the dynamics of the system with respect to the pure gravity case. The discussion of these cases is however beyond the limited purposes of this report, so we refer the reader to a future paper on the subject and concentrate now on the pure gravity case.

¿From the action (3) it is straightforward to obtain the equations of motion. They can be cast in a useful and simple form writing the twodimensional metric $h_{a b}$ in the "conformal gauge" [12]

$$
d s_{h}^{2} \equiv h_{a b} d x^{a} d x^{b}=4 \rho(u, v) d u d v
$$

The result is

$$
\begin{aligned}
& \partial_{u} \partial_{v} \phi-\alpha \rho e^{-\psi} \phi^{\beta}=0, \\
& \partial_{u} \partial_{v}(\ln \rho)-\alpha \beta \rho e^{-\psi} \phi^{\beta-1}-\gamma \partial_{u} \psi \partial_{v} \psi=0, \\
& \gamma \partial_{u}\left(\phi \partial_{v} \psi\right)+\gamma \partial_{v}\left(\phi \partial_{u} \psi\right)+\alpha \rho e^{-\psi} \phi^{\beta}=0, \\
& \rho \partial_{u}\left(\frac{\partial_{u} \phi}{\rho}\right)=\gamma \phi\left(\partial_{u} \psi\right)^{2}, \quad \rho \partial_{v}\left(\frac{\partial_{v} \phi}{\rho}\right)=\gamma \phi\left(\partial_{v} \psi\right)^{2},
\end{aligned}
$$


where $\alpha=q(q-1), \beta=-1 /(p+q)$, and $\gamma=-q(p+q) / 4 p$. The equations of motion (7-9) and the constraints (10) form a set of five non-linear and coupled second order partial differential equations in the unknown $\rho, \phi$, and $\psi$. However, as remarked in Ref. [12] only four of these equations are independent because Eqs. (7,8) and the constraints (10) imply (9), or, alternatively, Eqs. (7,9) imply (8).

Due to the nature of the system, the general solution of the equations of motion is very hard to find. However, it is possible to find the general static solution associated to the system of equations ( $7-10)$. According to Filippov [12] we define "static" those solutions of the equations of motion that can be cast in the form

$$
\psi \equiv \psi(\tau), \quad \phi \equiv \phi(\psi), \quad \rho \equiv \xi(\psi) \partial_{u} \tau \partial_{v} \tau
$$

where $\tau$ is a harmonic function: $\partial_{u} \partial_{v} \tau(u, v)=0$. The solutions (11) depend only on one coordinate $(\tau)$. This also implies that the metric coefficients in (2) depends on a single coordinate, and thus the geometry is static. In particular, since $\tau$ is harmonic we can write $\tau=U(u)+V(v)$, and from (6) we see that $U \mp V$ can be identified with the timelike coordinate and with the conformal radial coordinate in the line element (2) respectively. The arbitrariness in the choice of $U(u)$ and $V(v)$ reflects of course the invariance of (6) under coordinate reparametrization.

In the calculation of static solutions we are guided by the case of twodimensional pure dilaton - gravity models that has been proved to be completely integrable for any dilaton potential [12]. In that case it is also proved that all solutions are of the static type (extended Birkhoff theorem). In the present case we are only able to prove that the static sector is completely integrable and find the general static solution. It would then be worthwile to investigate if these solutions are also the general (static and non-static) solutions of the system, extending the Birkhoff theorem to the (black) $p$-branes (2).

Substituting (11) in the Eqs. (7-10), these reduce to the form

$$
\begin{aligned}
& \frac{d^{2} \phi}{d \psi^{2}} \psi^{\prime 2}+\frac{d \phi}{d \psi} \psi^{\prime \prime}-\alpha \xi e^{-\psi} \phi^{\beta}=0, \\
& \frac{d^{2}}{d \psi^{2}}(\ln \xi) \psi^{\prime 2}+\frac{d}{d \psi}(\ln \xi) \psi^{\prime \prime}-\alpha \beta \xi e^{-\psi} \phi^{\beta-1}=\gamma \psi^{\prime 2},
\end{aligned}
$$




$$
\begin{aligned}
& 2 \gamma \frac{d \phi}{d \psi} \psi^{\prime 2}+2 \gamma \phi \psi^{\prime \prime}+\alpha \xi e^{-\psi} \phi^{\beta}=0, \\
& \frac{d^{2} \phi}{d \psi^{2}} \psi^{\prime 2}+\frac{d \phi}{d \psi} \psi^{\prime \prime}-\frac{d \phi}{d \psi} \frac{d}{d \psi}(\ln \xi) \psi^{\prime 2}=\gamma \phi \psi^{\prime 2},
\end{aligned}
$$

where primes represent derivatives with respect to $\tau$. Using the equations of motion in the form above it is straighforward to obtain two first integrals and further reduce the system. After some algebra one obtains

$$
\begin{aligned}
& \phi \frac{d^{2} \phi}{d \tau^{2}}+a\left(\frac{d \phi}{d \tau}\right)^{2}+b \frac{d \phi}{d \tau}=c, \\
& \phi^{\prime}+2 \gamma \phi \psi^{\prime}=K \\
& \xi=\xi_{0} \phi^{1+\beta+\delta} e^{2 \gamma \delta \psi}, \\
& \frac{d^{2} \phi}{d \tau^{2}}=\alpha \xi_{0} \phi^{1+2 \beta+\delta} e^{(2 \gamma \delta-1) \psi},
\end{aligned}
$$

where $a=-(1+\beta+1 / 4 \gamma) \equiv(1-q) / q, b=-K(\delta-1 / 2 \gamma)$, and $c=K^{2} / 4 \gamma$. $\xi_{0}, K$, and $\delta$ are three constants of motion. The first one has no physical meaning and can be reabsorbed in the definition of $\tau$. The other two play a fundamental role in the geometry of the $p$-brane as we will see below. This set of equations can be read as follows: the integration of Eq. (16) gives $\phi$, the solution of Eq. (17) defines $\psi$ once $\phi$ is known, finally Eq. (18) defines the conformal part of the two-dimensional metric $\xi$ as a function of $\phi$ and $\psi$; Eq. (19) is dependent from the others and is used to determine the constants of integration.

\section{$3 \quad$ Static Black p-Branes}

The system of Eqs. (16-19), and in particular Eq. (16), is the starting point

to solve the system. Before looking for the general solution, let us calculate as a warming up exercise the boost symmetric solutions of Ref. [9]. 


\subsection{Boost Symmetric $p$-Branes}

In order to compare our results with those of Ref. [9] let us look for a solution for $\phi$ of the the form $\phi=r^{m} A(r)^{n}$, where $m$ and $n$ are real numbers to be determined by the equations of motion, and $r$ is related to $\tau$ by the relation $\tau=\int d r A(r)^{n-1}$. Choosing $\delta=-2 \beta$ (boost symmetric $p$-brane, see Eq. (22)) and after some algebra Eq. (16) can be solved and the system integrated. The final result is

$$
\begin{aligned}
& \phi=r^{q} A^{\frac{q(\Delta-1)}{2 \Delta(q-1)}}, \\
& e^{\psi}=r^{\frac{2 p}{p+q}} A^{\frac{p(\Delta-q)}{\Delta(q-1)(p+q)}}, \\
& \xi=\phi^{1+\frac{1}{p+q}} e^{-\frac{q}{p} \psi}, \quad q>1,
\end{aligned}
$$

where

$$
A(r)=1-\left(\frac{r_{+}}{r}\right)^{q-1},
$$

and $\Delta$ and $r_{+}$are two constants defined by the relations

$$
\Delta^{2}=q(p+1) /(p+q), \quad K=-\frac{q(q-1)}{2 \Delta} r_{+}^{q-1}
$$

Finally, the boost symmetric $p$-brane reads

$$
d s^{2}=A^{\frac{\Delta}{p+1}}\left(-d t^{2}+d z_{i}^{2}\right)+A^{\frac{2-q-\Delta}{q-1}} d r^{2}+r^{2} A^{\frac{1-\Delta}{q-1}} d \Omega^{(q)}
$$

Several interesting remarks can be suggested by the solution (25). First of all it is immediate to see that $K$ introduced in Eq. (17) is related to the radius of the horizon of the black $p$-brane (25). The role of the constant of motion $K$ is thus analog to the role of the mass in two-dimensional dilaton - gravity models, and in particular in the effective two-dimensional dilaton - gravity theory describing the Schwarzschild black hole. In those cases the existence of a functional conserved under time and space translations (the mass) is strictly related to the validity of the generalized Birkhoff theorem and to the dimensional collapse of the $1+1$ theory into a $0+1$ theory (see e.g. [13]). In the present case the existence of $K$ seems to suggest a similar conclusion, i.e. that all the solutions of the model described by (3) are actually static. Second, in (25) there are two different sets of solutions related by the sign

of $\Delta$. The (uncharged) boost symmetric $p$-branes of Ref. [9] correspond 
to the choice of positive $\Delta$. However, if one performs the discrete "dual" transformation $\Delta \rightarrow-\Delta$ other solutions are obtained. For instance in the case of the 5-brane in 10 dimensions we obtain the two solutions

$$
d s^{2}=\left(1-\frac{r_{+}^{2}}{r^{2}}\right)^{n_{1}}\left(-d t^{2}+d z_{i}^{2}\right)+\left(1-\frac{r_{+}^{2}}{r^{2}}\right)^{n_{2}} d r^{2}+r^{2}\left(1-\frac{r_{+}^{2}}{r^{2}}\right)^{n_{3}} d \Omega^{(3)}
$$

where $n \equiv(1 / 4,-5 / 4,-1 / 4)$ for $\Delta=3 / 2$ and $n \equiv(-1 / 4,1 / 4,5 / 4)$ for $\Delta=-3 / 2$. The "duality" invariance $\Delta \rightarrow-\Delta$ changes in particular the radius of the boost symmetric $p$-brane into its inverse. From the definition of $\Delta$ (24) it is straightforward to see that $\Delta^{2}>1$ for any value of $p$ and $q$ $\left(>1\right.$, of course). This implies that the singularity of the metric at $r=r_{+}$ has a divergent volume element for the set of solutions $\Delta>0$ and a finite volume element for the set with negative $\Delta$.

Finally, let us stress that the parameter $\delta$ defined in Eqs. (12-15) has been chosen equal to $-2 \beta$. Boost symmetric $p$-branes represent thus only a set of null measure in the space of static solutions.

\subsection{General Static Solution}

Let us now move to the calculation of the general static solution. We will see that the structure of the space of general static solutions is much richer that the space of the boost symmetric solutions.

The system (16-19) can be exacly integrated without introducing the semplifications used in the previous calculation. This program can be completed defining a new coordinate $x$ related to $\tau$ by the expression $\tau=\int \phi(x) d x$. After some algebra the general solution is found to be

$$
\begin{aligned}
& \phi=C_{\phi} e^{-\frac{K}{4 a \gamma}(1-2 \gamma \delta) x}(\sinh (K \bar{\Delta} x))^{\frac{1}{a}}, \\
& e^{\psi}=C_{\psi} e^{-\frac{K}{4 a \gamma}[\delta+2(1+\beta)] x}(\sinh (K \bar{\Delta} x))^{-\frac{1}{2 a \gamma}}, \\
& \xi=C_{\xi} e^{-\frac{K}{4 a \gamma}[\delta+(1+\beta)(1+2 \gamma \delta)] x}(\sinh (K \bar{\Delta} x))^{\frac{1+\beta}{a}},
\end{aligned}
$$

where $C_{\xi} C_{\psi}^{-1} C_{\phi}^{1+\beta}=x_{0}^{-2} \equiv K^{2} \bar{\Delta}^{2} /(q-1)^{2}$ and

$$
\bar{\Delta}^{2}=\frac{1}{4}\left[\delta\left(\delta-\frac{1}{\gamma}\right)-\frac{1+\beta}{\gamma}\right] \text {. }
$$


The metric of the $p$-brane reads then

$$
\begin{array}{r}
d s^{2}=-A_{1}^{2} e^{K \delta x} d t^{2}+A_{2}^{2} e^{-2 \beta K x} d z_{i}^{2}+e^{-\frac{K}{2 a \gamma}[1+2 \gamma \delta(\beta+1 / 4 \gamma)] x} \\
\cdot A_{3}^{2} \sinh ^{\frac{2}{a}}(K \bar{\Delta} x)\left[d x^{2}+x_{0}^{2} \sinh ^{2}(K \bar{\Delta} x) d \Omega^{(q)}\right]
\end{array}
$$

where $A_{1}^{2}=C_{\xi} C_{\phi}^{-(1+\beta)}, A_{2}^{2}=\left(C_{\xi} C_{\phi}^{1+\beta+1 / 2 \gamma} x_{0}^{2}\right)^{-4 \beta \gamma}, A_{3}^{2}=C_{\xi} C_{\phi}^{1-\beta}$, and the first two may be eventually reabsorbed in the redefinition of the time and $p$-brane coordinates. It is straightforward to check that, setting $\delta=-2 \beta$, (31) becomes the boost symmetric solution of Sec. 3.1. This can be easily proved using the coordinate transformation

$$
x=\frac{q}{2 K \Delta} \ln \left[1-\left(\frac{r_{+}}{r}\right)^{q-1}\right],
$$

and setting $A_{3}=2^{q /(1-q)} r_{+}^{q}$. In this case $x_{0}^{2}=4 r_{+}^{2(1-q)}, \bar{\Delta}^{2}=\Delta^{2} / q^{2}$, and the "duality" relation of the previous section is immediate since the metric is evidently invariant under change of sign of $\Delta$.

Starting form the line element (31), the geometrical properties of the general solution can be investigated for any value of the parameter $\delta$. This is beyond the scope of this short note, so we will not enter into details. Let us stress, however, that all solutions (31) are asymptotically flat for $x \rightarrow 0$, corresponding to $r \rightarrow \infty$ in the boost symmetric case of Sec. 3.1, for any value of the parameter $\delta$.

\section{Conclusions}

The effective two-dimensional dilaton - gravity - matter action (3) describes the dynamics of uncharged $p$-branes in $N \geq 4$ dimensions. In this paper we have proved that the model is completely integrable in the static sector and we have calculated the general solution. This result can be read from two different perpectives. First it is an example of an integrable model of twodimensional dilaton gravity plus matter (at least in the static sector) with a non trivial coupling between the fields, and the knowledge of exact solutions of complex theories is always welcome in theoretical physics. Second, the general solution of Sec. 3.2 describes the most general static uncharged black $p$-brane in $N \geq 4$ dimensions. The existence of the general static solution of 
Sec. 3.2, and its relation to the two-dimensional theory (3), imply that some essential properties of (uncharged) $p$-branes are very similar to the properties of solutions of other well-known dilaton - gravity models, as for instance the spherically simmetric reduced pure gravity. Among these properties, the definition of the $p$-brane mass in canonical form and the extension of the Birkhoff theorem to this class of theories (see e.g. [13]), are certainly worth being explored in the future.

\section{Acknowledgements}

This work was in part supported by the foreign grant N. 3229/96 of the University of Torino and by the Angelo Della Riccia Foundation, Florence, Italy. We are very grateful to Ruth Gregory, Paulo Moniz, Alex Vilenkin, and, in particular, to Vittorio de Alfaro for interesting discussions and useful suggestions on various questions connected to the subject of this paper.

\section{References}

[1] It is very difficult to give a detailed and complete bibliography covering all aspects of this subject. The references cited below are intended to be nor a complete nor an exhaustive review of the present research in classical and quantum gravity in generic $N$ dimensions. For a full discussion on the many aspects of this subject the reader is referred to the excellent recent reviews cited in the introduction. Other references about the most advanced research can also be found in the recent paper by V.D. Ivashchuk, V.N. Melnikov, and M. Rainer [2] and in several contributions to the Proceedings of the 2nd Conference on Constrained Dynamics and Quantum Gravity, Santa Margherita Ligure, September 1996 (Proc. Supplement, Nucl. Phys. B, in press, 1997, eds. V. de Alfaro et al.. We apologize in advance for any omission.

[2] V.D. Ivashchuk, V.N. Melnikov, and M. Rainer, "Multidimensional $\sigma$-models with composite electric $p$-branes", e-Print Archive: grqc/9705005 and references therein.

[3] E. Witten, Phys. Rev. D44, 314 (1991). 
[4] H. Kastrup and T. Thiemann, Nucl. Phys. B425, 665 (1994); D. Marolf, Phys. Rev. D53, 6979 (1996); K.V. Kuchař, Phys. Rev. D50, 3961 (1994).

[5] C. Callan, S. Giddings, J. Harvey, and A. Strominger, Phys. Rev. D45, 1005 (1992); J. Russo, L. Susskind, and L. Thorlacius, Phys. Rev. D46, 3444 (1992); S. Carlip, J. Gegenberg, and R.B. Mann, Phys. Rev. D51, 6854 (1995); A. Barvinskii and G. Kunstatter, Phys. Lett. B389, 231 (1996) and references therein.

[6] See for instance: H. Nicolai, D. Korotkin, and H. Samtleben, "Integrable Classical and Quantum Gravity", Lectures given at NATO Advanced Study Institute on Quantum Fields and Quantum Space Time, Cargèse, France, 22 July - 3 August.

[7] E. Benedict, R. Jackiw, and H.-J. Lee, Phys. Rev. D54, 6213 (1996); D. Cangemi, R. Jackiw, and B. Zwiebach, Ann. Physics (N.Y.) 245, 408 (1995); D. Cangemi and R. Jackiw, Phys. Rev. Lett. 69, 233 (1992); K.V. Kuchař, J.D. Romano, and M. Varadarajan, Phys. Rev. D55, 795 (1997); D. Louis-Martinez, J.. Gegenberg, and G. Kunstatter, Phys. Lett. B321, 193 (1994) and references therein.

[8] K.A. Bronnikov and V.N. Melnikov, Ann. Phys. 239, 40 (1995); V.D. Ivashchuk and V.N. Melnikov, Theor. Math. Phys. 98, 212 (1994).

[9] R. Gregory, Nucl. Phys. B467, 159 (1996).

[10] K.S. Stelle, "Lectures on Supergravity p-branes", Lectures presented at 1996 ICTP Summer School, Trieste, Italy.

[11] M.J. Duff, H. Lu, C.N. Pope, Phys. Lett. B382, 73 (1996); M.J. Duff, Ramzi R. Khuri, J.X. Lu, Phys. Rep. 259, 213 (1995).

[12] A.T. Filippov, Mod. Phys. Lett. A11, 1691 (1996); Int. J. Mod. Phys. A12, 13 (1997).

[13] M. Cavaglià, V. de Alfaro and A.T. Filippov, "The Birkhoff Theorem in the Quantum Theory of Two-Dimensional Dilaton Gravity", Report No. DFTT 20/97, e-Print Archive: hep-th/9704164. 